Bulletin of the Section of Logic

Volume 50/1 (2021), pp. 97-123

https://doi.org/10.18778/0138-0680.2020.28

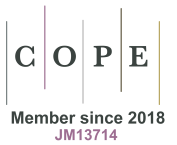

Rajab Ali Borzooei

Gholam Reza Rezaei

Mona Aaly Kologani

Young Bae Jun

\title{
SOJU FILTERS IN HOOP ALGEBRAS
}

\begin{abstract}
The notions of (implicative) soju filters in a hoop algebra are introduced, and related properties are investigated. Relations between a soju sub-hoop, a soju filter and an implicative soju filter are discussed. Conditions for a soju filter to be implicative are displayed, and characterizations of an implicative soju filters are considered. The extension property of an implicative soju filter is established.
\end{abstract}

Keywords: Soju sub-hoop, soju filter, implicative soju filter.

2010 Mathematical Subject Classification: 06F35, 03G25, 08A72.

\section{Introduction}

It is well-known that an intuitionistic fuzzy set is a generalization of a fuzzy set, and it is introduced by Attanassov [1]. Molodtsov [15] introduced the concept of soft set as a new mathematical tool for dealing with uncertainties. For more information on intuitionistic fuzzy sets and soft sets, see $[1,2,14,15]$. By combining intuitionistic fuzzy set and soft set, Jun et al. [11] introduced a new structure, so called soju structure, and they applied it to BCK/BCI-algebras. Xin et al. [16] introduced the notion of positive implicative soju ideal in BCK-algebra, and investigate related properties. We discuss relations between soju ideal and positive implicative soju ideal,

Presented by: Jie Fang

Received: July 27, 2019

Published online: December 30, 2020

(C) Copyright for this edition by Uniwersytet Łódzki, Łódź 2020 
and established characterizations of positive implicative soju ideal. They constructed extension property for positive implicative soju ideal.

In this article, we apply the soju structure to hoop algebras, which is introduced by Bosbach in $[8,9]$ and studied $[12,4,3,5,6,7,16]$. We introduce the concepts of soju sub-hoops and (implicative) soju filters in a hoop algebra, and investigate related properties. We discuss the homomorphic preimage and image of soju sub-hoops. We consider relations between a soju sub-hoop, a soju filter and an implicative soju filter. We provide conditions for a soju filter to be implicative, and characterize an implicative soju filter. We establish the extension property of an implicative soju filter.

\section{Preliminaries}

By a hoop (or hoop algebra) we mean an algebra $(H, \odot, \rightarrow, 1)$ in which $(H, \odot, 1)$ is a commutative monoid and the following assertions are valid.

(H1) $x \rightarrow x=1$,

(H2) $x \odot(x \rightarrow y)=y \odot(y \rightarrow x)$,

(H3) $x \rightarrow(y \rightarrow z)=(x \odot y) \rightarrow z$

for all $x, y, z \in H$. We define a relation " $\leq$ " on a hoop $H$ by

$$
(\forall x, y \in H)(x \leq y \Leftrightarrow x \rightarrow y=1) .
$$

It is easy to see that $(H, \leq)$ is a poset.

A nonempty subset $S$ of a hoop algebra $H$ is called a sub-hoop of $H$ if it satisfies:

$$
(\forall x, y \in S)(x \odot y \in S, x \rightarrow y \in S) .
$$

Note that every sub-hoop contains the element 1.

Proposition $2.1([10])$. Let $(H, \odot, \rightarrow, 1)$ be a hoop algebra. For any $x, y, z \in H$, the following conditions hold:

(a1) $(H, \leq)$ is a meet-semilattice with $x \wedge y=x \odot(x \rightarrow y)$,

(a2) $x \odot y \leq z$ if and anly if $x \leq y \rightarrow z$,

(a3) $x \odot y \leq x, y$ and $x^{n} \leq x$ for any $n \in \mathbb{N}$, 
(a4) $x \leq y \rightarrow x$,

(a5) $1 \rightarrow x=x$ and $x \rightarrow 1=1$,

$(a 6) x \odot(x \rightarrow y) \leq y, x \odot y \leq x \wedge y \leq x \rightarrow y$,

(a7) $x \rightarrow y \leq(y \rightarrow z) \rightarrow(x \rightarrow z)$,

(a8) $x \leq y$ implies $x \odot z \leq y \odot z, z \rightarrow x \leq z \rightarrow y$ and $y \rightarrow z \leq x \rightarrow z$, $(a 9) x \rightarrow(y \rightarrow z)=y \rightarrow(x \rightarrow z)$.

A nonempty subset $F$ of a hoop algebra $H$ is called

- a filter of $H$ (see [10]) if the following assertions are valid.

$$
\begin{aligned}
& (\forall x, y \in H)(x, y \in F \Rightarrow x \odot y \in F), \\
& (\forall x, y \in H)(x \in F, x \leq y \Rightarrow y \in F) .
\end{aligned}
$$

- an implicative filter of $H$ (see [13]) if the following assertions are valid.

$$
\begin{aligned}
& 1 \in F, \\
& (\forall x, y, z \in H)(x \rightarrow((y \rightarrow z) \rightarrow y) \in F, x \in F \Rightarrow y \in F) .
\end{aligned}
$$

Note that the conditions (2.3) and (2.4) means that $F$ is closed under the operation $\odot$ and $F$ is upward closed, respectively.

Note that a subset $F$ of a hoop algebra $H$ is a filter of $H$ if and only if $F$ satisfies the condition (2.5) and

$$
(\forall x, y \in H)(x \rightarrow y \in F, x \in F \Rightarrow y \in F) .
$$

For more information on intuitionistic fuzzy sets and soft sets, see [4], [3] and [16].

\section{Soju sub-hoops and soju filters}

In what follows, let $U$ be an initial universe set unless otherwise specified.

Definition 3.1 ([11]). Let $E$ be a set of parameters. For any subset $A$ of $E$, let $\sigma:=\left(\mu_{\sigma}, \gamma_{\sigma}\right)$ be an intuitionistic fuzzy set in $A$ and $(\tilde{F}, A)$ be 
a soft set over $U$. Then a pair $(A,\langle\sigma ; \tilde{F}\rangle)$ is called a soju structure over $([0,1], U)$.

Given a soju structure $(E,\langle\sigma ; \tilde{F}\rangle)$ over $([0,1], U), \alpha \in 2^{U}$ and $(t, s) \in$ $[0,1] \times[0,1]$ with $t+s \leq 1$, consider the following sets:

$$
\begin{aligned}
& U\left(\mu_{\sigma} ; t\right):=\left\{x \in E \mid \mu_{\sigma}(x) \geq t\right\}, \\
& L\left(\gamma_{\sigma} ; s\right):=\left\{x \in E \mid \gamma_{\sigma}(x) \leq s\right\}, \\
& i(\tilde{F} ; \alpha):=\{x \in E \mid \tilde{F}(x) \supseteq \alpha\},
\end{aligned}
$$

which are called soju level sets of $(E,\langle\sigma ; \tilde{F}\rangle)$.

Definition 3.2. Let $A$ be a subset of a hoop algebra $E$. A soju structure $(A,\langle\sigma ; \tilde{F}\rangle)$ over $([0,1], U)$ is called a soju sub-hoop based on $A$ (briefly, soju $A$-sub-hoop) of $E$ if the following condition is valid.

$$
(\forall x, y \in A)\left(x \bullet y \in A \Rightarrow\left\{\begin{array}{l}
\mu_{\sigma}(x \bullet y) \geq \min \left\{\mu_{\sigma}(x), \mu_{\sigma}(y)\right\} \\
\gamma_{\sigma}(x \bullet y) \leq \max \left\{\gamma_{\sigma}(x), \gamma_{\sigma}(y)\right\} \\
\tilde{F}(x \bullet y) \supseteq \tilde{F}(x) \cap \tilde{F}(y)
\end{array}\right)\right.
$$

for $\bullet \in\{\odot, \rightarrow\}$.

Example 3.3. Consider a hoop algebra $(E, \odot, \rightarrow, 1)$ in which $E=\{0, a, b, 1\}$ with binary operations $\rightarrow$ and $\odot$ which are given as follows:

\begin{tabular}{c|ccccc|cccc}
$\rightarrow$ & 0 & $a$ & $b$ & 1 & $\odot$ & 0 & $a$ & $b$ & 1 \\
\hline 0 & 1 & 1 & 1 & 1 & 0 & 0 & 0 & 0 & 0 \\
$a$ & $a$ & 1 & 1 & 1 & $a$ & 0 & 0 & $a$ & $a$ \\
$b$ & 0 & $a$ & 1 & 1 & $b$ & 0 & $a$ & $b$ & $b$ \\
1 & 0 & $a$ & $b$ & 1 & 1 & 0 & $a$ & $b$ & 1
\end{tabular}

(1) The set $A=\{0, a, 1\}$ is a sub-hoop of $E$. Define a soju structure $(A,\langle\sigma ; \tilde{F}\rangle)$ over $([0,1], U)=([0,1], \mathbb{Z})$ by Table 1 .

By routine calculations, we know that $(A,\langle\sigma ; \tilde{F}\rangle)$ is a soju $A$-sub-hoop of $E$.

(2) Define a soju structure $(E,\langle\sigma ; \tilde{F}\rangle)$ over $([0,1], U)=([0,1], \mathbb{Z})$ by Table 2 .

It is routine to verify that $(E,\langle\sigma ; \tilde{F}\rangle)$ is a soju sub-hoop of $E$.

Proposition 3.4. Let $A$ be a sub-hoop of a hoop algebra $E$. Every soju $A$-sub-hoop $(A,\langle\sigma ; \tilde{F}\rangle)$ of $E$ satisfies the following condition. 
Table 1. Tabular representation of $(A,\langle\sigma ; \tilde{F}\rangle)$

\begin{tabular}{c||c|c}
\hline$A$ & $\sigma(x)=\left(\mu_{\sigma}(x), \gamma_{\sigma}(x)\right)$ & $\tilde{F}(x)$ \\
\hline 0 & $(0.75,0.2)$ & $4 \mathbb{Z}$ \\
$a$ & $(0.45,0.5)$ & $4 \mathbb{N}$ \\
1 & $(0.85,0.1)$ & $2 \mathbb{Z}$ \\
\hline
\end{tabular}

Table 2. Tabular representation of $(E,\langle\sigma ; \tilde{F}\rangle)$

\begin{tabular}{c||c|c}
\hline$E$ & $\sigma(x)=\left(\mu_{\sigma}(x), \gamma_{\sigma}(x)\right)$ & $\tilde{F}(x)$ \\
\hline 0 & $(0.4,0.6)$ & $2 \mathbb{Z}$ \\
$a$ & $(0.4,0.5)$ & $6 \mathbb{N}$ \\
$b$ & $(0.6,0.3)$ & $3 \mathbb{Z}$ \\
1 & $(0.8,0.1)$ & $\mathbb{Z}$ \\
\hline
\end{tabular}

$$
(\forall x \in A)\left(\mu_{\sigma}(1) \geq \mu_{\sigma}(x), \gamma_{\sigma}(1) \leq \gamma_{\sigma}(x), \tilde{F}(1) \supseteq \tilde{F}(x)\right),
$$

Proof: Since $x \rightarrow x=1$ for all $x \in E$, it is straightforward by (3.1).

TheOREM 3.5. Given a hoop algebra $E$, the soju structure $(E,\langle\sigma ; \tilde{F}\rangle)$ over $([0,1], U)$ is a soju sub-hoop of $E$ if and only if its nonempty soju level sets $U\left(\mu_{\sigma} ; t\right), L\left(\gamma_{\sigma} ; s\right)$ and $i(\tilde{F} ; \alpha)$ are sub-hoops of $E$ for all $\alpha \in 2^{U}$ and $(t, s) \in[0,1] \times[0,1]$ with $t+s \leq 1$.

Proof: Assume that $(E,\langle\sigma ; \tilde{F}\rangle)$ is a soju sub-hoop of $E$ and let $\alpha \in 2^{U}$ and $(t, s) \in[0,1] \times[0,1]$ be such that $t+s \leq 1$, and $U\left(\mu_{\sigma} ; t\right), L\left(\gamma_{\sigma} ; s\right)$ and $i(\tilde{F} ; \alpha)$ are non-empty. Let $x, y \in E$ be such that $x, y \in U\left(\mu_{\sigma} ; t\right) \cap L\left(\gamma_{\sigma} ; s\right) \cap$ $i(\tilde{F} ; \alpha)$. Then $\mu_{\sigma}(x) \geq t, \mu_{\sigma}(y) \geq t, \gamma_{\sigma}(x) \leq s, \gamma_{\sigma}(y) \leq s, \tilde{F}(x) \supseteq \alpha$ and $\tilde{F}(y) \supseteq \alpha$. It follows from (3.1) that

$$
\begin{aligned}
& \mu_{\sigma}(x \bullet y) \geq \min \left\{\mu_{\sigma}(x), \mu_{\sigma}(y)\right\} \geq t, \\
& \gamma_{\sigma}(x \bullet y) \leq \max \left\{\gamma_{\sigma}(x), \gamma_{\sigma}(y)\right\} \leq s, \\
& \tilde{F}(x \bullet y) \supseteq \tilde{F}(x) \cap \tilde{F}(y) \supseteq \alpha
\end{aligned}
$$

for $\bullet \in\{\odot, \rightarrow\}$. Hence $x \bullet y \in U\left(\mu_{\sigma} ; t\right) \cap L\left(\gamma_{\sigma} ; s\right) \cap i(\tilde{F} ; \alpha)$, and therefore $U\left(\mu_{\sigma} ; t\right), L\left(\gamma_{\sigma} ; s\right)$ and $i(\tilde{F} ; \alpha)$ are sub-hoops of $E$. 
Conversely, suppose that the nonempty soju level sets $U\left(\mu_{\sigma} ; t\right), L\left(\gamma_{\sigma} ; s\right)$ and $i(\tilde{F} ; \alpha)$ of $(E,\langle\sigma ; \tilde{F}\rangle)$ are sub-hoops of $E$ for all $\alpha \in 2^{U}$ and $(t, s) \in$ $[0,1] \times[0,1]$ with $t+s \leq 1$. For any $x, y \in E$, let $t_{x}, t_{y}, s_{x}, s_{y} \in[0,1]$ and $\alpha_{x}, \alpha_{y} \in 2^{U}$ be such that $\mu_{\sigma}(x)=t_{x}, \mu_{\sigma}(y)=t_{y}, \gamma_{\sigma}(x)=s_{x}, \gamma_{\sigma}(y)=s_{y}$, $\tilde{F}(x)=\alpha_{x}$ and $\tilde{F}(y)=\alpha_{y}$. If we take $t:=\min \left\{t_{x}, t_{y}\right\}, s:=\max \left\{s_{x}, s_{y}\right\}$ and $\alpha:=\alpha_{x} \cap \alpha_{y}$, then $x, y \in U\left(\mu_{\sigma} ; t\right) \cap L\left(\gamma_{\sigma} ; s\right) \cap i(\tilde{F} ; \alpha)$. Thus $x \bullet y \in$ $U\left(\mu_{\sigma} ; t\right) \cap L\left(\gamma_{\sigma} ; s\right) \cap i(\tilde{F} ; \alpha)$, and so

$$
\begin{aligned}
& \mu_{\sigma}(x \bullet y) \geq t=\min \left\{t_{x}, t_{y}\right\}=\min \left\{\mu_{\sigma}(x), \mu_{\sigma}(y)\right\}, \\
& \gamma_{\sigma}(x \bullet y) \leq s=\max \left\{s_{x}, s_{y}\right\}=\max \left\{\gamma_{\sigma}(x), \gamma_{\sigma}(y)\right\}, \\
& \tilde{F}(x \bullet y) \supseteq \alpha=\alpha_{x} \cap \alpha_{y}=\tilde{F}(x) \cap \tilde{F}(y)
\end{aligned}
$$

for $\bullet \in\{\odot, \rightarrow\}$. Therefore $(E,\langle\sigma ; \tilde{F}\rangle)$ is a soju sub-hoop of $E$.

We define the image and preimage of soju structures. Let $E$ and $K$ be nonempty sets and $f: E \rightarrow K$ be a mapping.

Definition 3.6. (1) If $(K,\langle\tau ; \tilde{G}\rangle)$ is a soju structure over $([0,1], U)$, then the preimage of $(K,\langle\tau ; \tilde{G}\rangle)$ under $f$ is denoted by $f^{-1}(K,\langle\tau ; \tilde{G}\rangle)$ and is defined to be a soju structure $(E,\langle\sigma ; \tilde{F}\rangle)$ over $([0,1], U)$ with $\mu_{\sigma}=f^{-1}\left(\mu_{\tau}\right)$, $\gamma_{\sigma}=f^{-1}\left(\gamma_{\tau}\right)$ and

$$
\tilde{F}=f^{-1}(\tilde{G})=\left\{\left(x, f^{-1}(\tilde{G})(x)\right) \mid x \in E, f^{-1}(\tilde{G})(x) \in 2^{U}\right\}
$$

where $\mu_{\sigma}(x)=f^{-1}\left(\mu_{\tau}\right)(x)=\mu_{\tau}(f(x)), \gamma_{\sigma}(x)=f^{-1}\left(\gamma_{\tau}\right)(x)=\gamma_{\tau}(f(x))$, and $f^{-1}(\tilde{G})(x)=\tilde{G}(f(x))$ for all $x \in E$.

(2) If $(E,\langle\sigma ; \tilde{F}\rangle)$ is a soju structure over $([0,1], U)$, then the image of $(E,\langle\sigma ; \tilde{F}\rangle)$ under $f$ is denoted by $f(E,\langle\sigma ; \tilde{F}\rangle)$ and is defined to be a soju structure $(K,\langle\tau ; \tilde{G}\rangle)$ over $([0,1], U)$ with $\mu_{\tau}=f\left(\mu_{\sigma}\right), \gamma_{\tau}=f\left(\gamma_{\sigma}\right)$ and $\tilde{G}=f(\tilde{F})$ where

$$
\begin{aligned}
& \mu_{\tau}(y)=f\left(\mu_{\sigma}\right)(y)= \begin{cases}\sup _{x \in f^{-1}(y)} \mu_{\sigma}(x) & \text { if } f^{-1}(y) \neq \emptyset, \\
0 & \text { otherwise, }\end{cases} \\
& \gamma_{\tau}(y)=f\left(\gamma_{\sigma}\right)(y)= \begin{cases}\inf _{x \in f^{-1}(y)} \gamma_{\sigma}(x) & \text { if } f^{-1}(y) \neq \emptyset, \\
1 & \text { otherwise, }\end{cases}
\end{aligned}
$$

and 


$$
\tilde{G}(y)=f(\tilde{F})(y)= \begin{cases}\bigcup_{x \in f^{-1}(y)} \tilde{F}(x) & \text { if } f^{-1}(y) \neq \emptyset \\ \emptyset & \text { otherwise }\end{cases}
$$

for all $y \in K$.

TheOREM 3.7. Let $f: E \rightarrow K$ be a homomorphism of hoop algebras and let $(K,\langle\tau ; \tilde{G}\rangle)$ be a soju structure over $([0,1], U)$. If $(K,\langle\tau ; \tilde{G}\rangle)$ is a soju sub-hoop of $K$, then the preimage of $(K,\langle\tau ; \tilde{G}\rangle)$ under $f$ is a soju sub-hoop of $E$.

Proof: For any $\bullet \in\{\odot, \rightarrow\}$ and any $x_{1}, x_{2} \in E$, we have

$$
\begin{aligned}
\mu_{\sigma}\left(x_{1} \bullet x_{2}\right) & =f^{-1}\left(\mu_{\tau}\right)\left(x_{1} \bullet x_{2}\right)=\mu_{\tau}\left(f\left(x_{1} \bullet x_{2}\right)\right) \\
& =\mu_{\tau}\left(f\left(x_{1}\right) \bullet f\left(x_{2}\right)\right) \\
& \geq \min \left\{\mu_{\tau}\left(f\left(x_{1}\right)\right), \mu_{\tau}\left(f\left(x_{2}\right)\right)\right\} \\
& =\min \left\{f^{-1}\left(\mu_{\tau}\right)\left(x_{1}\right), f^{-1}\left(\mu_{\tau}\right)\left(x_{2}\right)\right\} \\
& =\min \left\{\mu_{\sigma}\left(x_{1}\right), \mu_{\sigma}\left(x_{2}\right)\right\}, \\
\gamma_{\sigma}\left(x_{1} \bullet x_{2}\right) & =f^{-1}\left(\gamma_{\tau}\right)\left(x_{1} \bullet x_{2}\right)=\gamma_{\tau}\left(f\left(x_{1} \bullet x_{2}\right)\right) \\
& =\gamma_{\tau}\left(f\left(x_{1}\right) \bullet f\left(x_{2}\right)\right) \\
& \leq \max \left\{\gamma_{\tau}\left(f\left(x_{1}\right)\right), \gamma_{\tau}\left(f\left(x_{2}\right)\right)\right\} \\
& =\max \left\{f^{-1}\left(\gamma_{\tau}\right)\left(x_{1}\right), f^{-1}\left(\gamma_{\tau}\right)\left(x_{2}\right)\right\} \\
& =\max \left\{\gamma_{\sigma}\left(x_{1}\right), \gamma_{\sigma}\left(x_{2}\right)\right\},
\end{aligned}
$$

and

$$
\begin{aligned}
\tilde{F}\left(x_{1} \bullet x_{2}\right) & =f^{-1}(\tilde{G})\left(x_{1} \bullet x_{2}\right)=\tilde{G}\left(f\left(x_{1} \bullet x_{2}\right)\right) \\
& =\tilde{G}\left(f\left(x_{1}\right) \bullet f\left(x_{2}\right)\right) \\
& \supseteq \tilde{G}\left(f\left(x_{1}\right)\right) \cap \tilde{G}\left(f\left(x_{2}\right)\right) \\
& =f^{-1}(\tilde{G})\left(x_{1}\right) \cap f^{-1}(\tilde{G})\left(x_{2}\right) \\
& =\tilde{F}\left(x_{1}\right) \cap \tilde{F}\left(x_{2}\right) .
\end{aligned}
$$

Therefore $(E,\langle\sigma ; \tilde{F}\rangle)=f^{-1}(K,\langle\tau ; \tilde{G}\rangle)$ is a soju sub-hoop of $E$.

TheOrem 3.8. Let $f: E \rightarrow K$ be a homomorphism of hoop algebras and let $(E,\langle\sigma ; \tilde{F}\rangle)$ be a soju structure over $([0,1], U)$. If $(E,\langle\sigma ; \tilde{F}\rangle)$ is a soju sub-hoop of $E$ and $f$ is injective, then the image of $(E,\langle\sigma ; \tilde{F}\rangle)$ under $f$ is a soju sub-hoop of $K$. 
Proof: Let $y_{1}, y_{2} \in K$ and $\bullet \in\{\odot, \rightarrow\}$. If at least one of $f^{-1}\left(y_{1}\right)$ and $f^{-1}\left(y_{2}\right)$ is empty, then it is clear that

$$
\begin{aligned}
& \mu_{\tau}\left(y_{1} \bullet y_{2}\right) \geq \min \left\{\mu_{\tau}\left(y_{1}\right), \mu_{\tau}\left(y_{2}\right)\right\} \\
& \gamma_{\tau}\left(y_{1} \bullet y_{2}\right) \leq \max \left\{\gamma_{\tau}\left(y_{1}\right), \gamma_{\tau}\left(y_{2}\right)\right\}
\end{aligned}
$$

and $\tilde{G}\left(y_{1} \bullet y_{2}\right) \supseteq \tilde{G}\left(y_{1}\right) \cap \tilde{G}\left(y_{2}\right)$. Assume that $f^{-1}\left(y_{1}\right)$ and $f^{-1}\left(y_{2}\right)$ are nonempty. Then

$$
\begin{aligned}
& \min \left\{\mu_{\tau}\left(y_{1}\right), \mu_{\tau}\left(y_{2}\right)\right\}=\min \left\{f\left(\mu_{\sigma}\right)\left(y_{1}\right), f\left(\mu_{\sigma}\right)\left(y_{2}\right)\right\} \\
& =\min \left\{\sup _{x_{1} \in f^{-1}\left(y_{1}\right)} \mu_{\sigma}\left(x_{1}\right), \sup _{x_{2} \in f^{-1}\left(y_{2}\right)} \mu_{\sigma}\left(x_{2}\right)\right\} \\
& =\sup _{\substack{x_{1} \in f^{-1}\left(y_{1}\right) \\
x_{2} \in f^{-1}\left(y_{2}\right)}} \min \left\{\mu_{\sigma}\left(x_{1}\right), \mu_{\sigma}\left(x_{2}\right)\right\} \\
& \leq \sup _{\substack{x_{1} \in f^{-1}\left(y_{1}\right) \\
x_{2} \in f^{-1}\left(y_{2}\right)}} \mu_{\sigma}\left(x_{1} \bullet x_{2}\right) \\
& =\sup _{x \in f^{-1}\left(y_{1} \bullet y_{2}\right)} \mu_{\sigma}(x) \\
& =f\left(\mu_{\sigma}\right)\left(y_{1} \bullet y_{2}\right)=\mu_{\tau}\left(y_{1} \bullet y_{2}\right), \\
& \max \left\{\gamma_{\tau}\left(y_{1}\right), \gamma_{\tau}\left(y_{2}\right)\right\}=\max \left\{f\left(\gamma_{\sigma}\right)\left(y_{1}\right), f\left(\gamma_{\sigma}\right)\left(y_{2}\right)\right\} \\
& =\max \left\{\inf _{x_{1} \in f^{-1}\left(y_{1}\right)} \gamma_{\sigma}\left(x_{1}\right), \inf _{x_{2} \in f^{-1}\left(y_{2}\right)} \gamma_{\sigma}\left(x_{2}\right)\right\} \\
& =\inf _{\substack{x_{1} \in f^{-1}\left(y_{1}\right) \\
x_{2} \in f^{-1}\left(y_{2}\right)}} \max \left\{\gamma_{\sigma}\left(x_{1}\right), \gamma_{\sigma}\left(x_{2}\right)\right\} \\
& \geq \inf _{\substack{x_{1} \in f^{-1}\left(y_{1}\right) \\
x_{2} \in f^{-1}\left(y_{2}\right)}} \gamma_{\sigma}\left(x_{1} \bullet x_{2}\right) \\
& =\inf _{x \in f^{-1}\left(y_{1} \bullet y_{2}\right)} \gamma_{\sigma}(x) \\
& =f\left(\gamma_{\sigma}\right)\left(y_{1} \bullet y_{2}\right)=\gamma_{\tau}\left(y_{1} \bullet y_{2}\right) \text {, }
\end{aligned}
$$

and 


$$
\begin{aligned}
& \tilde{G}\left(y_{1}\right) \cap \tilde{G}\left(y_{2}\right)=f\left(\tilde{F}\left(y_{1}\right)\right) \cap f\left(\tilde{F}\left(y_{2}\right)\right) \\
& =\left(\bigcup_{x_{1} \in f^{-1}\left(y_{1}\right)} \tilde{F}\left(x_{1}\right)\right) \cap\left(\bigcup_{x_{2} \in f^{-1}\left(y_{2}\right)} \tilde{F}\left(x_{2}\right)\right) \\
& =\bigcup_{\substack{x_{1} \in f^{-1}\left(y_{1}\right) \\
x_{2} \in f^{-1}\left(y_{2}\right)}}\left(\tilde{F}\left(x_{1}\right) \cap \tilde{F}\left(x_{2}\right)\right) \\
& \subseteq \bigcup_{\substack{x_{1} \in f^{-1}\left(y_{1}\right) \\
x_{2} \in f^{-1}\left(y_{2}\right)}} \tilde{F}\left(x_{1} \bullet x_{2}\right) \\
& =\bigcup_{x \in f^{-1}\left(y_{1} \bullet y_{2}\right)} \tilde{F}(x) \\
& =f(\tilde{F})\left(y_{1} \bullet y_{2}\right)=\tilde{G}\left(y_{1} \bullet y_{2}\right) .
\end{aligned}
$$

Therefore $(K,\langle\tau ; \tilde{G}\rangle)$, the image of $(E,\langle\sigma ; \tilde{F}\rangle)$ under $f$, is a soju sub-hoop of $K$.

Definition 3.9. Let $A$ be a subset of a hoop algebra $E$. A soju structure $(A,\langle\sigma ; \tilde{F}\rangle)$ over $([0,1], U)$ is called a soju filter based on $A$ (briefly, soju $A$-filter $)$ of $E$ if the following condition is valid.

$$
\begin{aligned}
& (\forall x, y \in A)\left(x \odot y \in A \Rightarrow\left\{\begin{array}{l}
\mu_{\sigma}(x \odot y) \geq \min \left\{\mu_{\sigma}(x), \mu_{\sigma}(y)\right\} \\
\gamma_{\sigma}(x \odot y) \leq \max \left\{\gamma_{\sigma}(x), \gamma_{\sigma}(y)\right\} \\
\tilde{F}(x \odot y) \supseteq \tilde{F}(x) \cap \tilde{F}(y)
\end{array}\right)\right. \\
& (\forall x, y \in A)\left(x \leq y \Rightarrow\left\{\begin{array}{l}
\mu_{\sigma}(x) \leq \mu_{\sigma}(y), \\
\gamma_{\sigma}(x) \geq \gamma_{\sigma}(y) \\
\tilde{F}(x) \subseteq \tilde{F}(y)
\end{array}\right) .\right.
\end{aligned}
$$

A soju $E$-filter is simply called a soju filter.

Example 3.10. Consider the hoop algebra $(E, \odot, \rightarrow, 1)$ in Example 3.3. Define a soju structure $(E,\langle\sigma ; \tilde{F}\rangle)$ over $([0,1], U)=([0,1], \mathbb{Z})$ by Table 3 . It is routine to verify that $(E,\langle\sigma ; \tilde{F}\rangle)$ is a soju filter of $E$. 
Table 3. Tabular representation of $(E,\langle\sigma ; \tilde{F}\rangle)$

\begin{tabular}{c||c|c}
\hline$E$ & $\sigma(x)=\left(\mu_{\sigma}(x), \gamma_{\sigma}(x)\right)$ & $\tilde{F}(x)$ \\
\hline 0 & $(0.3,0.6)$ & $3 \mathbb{N}$ \\
$a$ & $(0.3,0.5)$ & $3 \mathbb{N}$ \\
$b$ & $(0.6,0.3)$ & $3 \mathbb{Z}$ \\
1 & $(0.7,0.1)$ & $\mathbb{Z}$ \\
\hline
\end{tabular}

TheOrem 3.11. Let $A$ be a sub-hoop of a hoop algebra $E$. Then a soju structure $(A,\langle\sigma ; \tilde{F}\rangle)$ over $([0,1], U)$ is a soju $A$-filter of $E$ if and only if it satisfies (3.2) and

$$
(\forall x, y \in A)\left(\begin{array}{l}
\mu_{\sigma}(y) \geq \min \left\{\mu_{\sigma}(x), \mu_{\sigma}(x \rightarrow y)\right\} \\
\gamma_{\sigma}(y) \leq \max \left\{\gamma_{\sigma}(x), \gamma_{\sigma}(x \rightarrow y)\right\} \\
\tilde{F}(y) \supseteq \tilde{F}(x) \cap \tilde{F}(x \rightarrow y)
\end{array}\right) .
$$

Proof: Let $(A,\langle\sigma ; \tilde{F}\rangle)$ be a soju $A$-filter of $E$. Since $x \leq 1$ for all $x \in E$, it follows from (3.4) that we have (3.2). For any $x, y \in A$, we get $x \odot(x \rightarrow$ $y) \leq y$. Using (3.3) and (3.4), we have

$$
\begin{aligned}
& \mu_{\sigma}(y) \geq \mu_{\sigma}(x \odot(x \rightarrow y)) \geq \min \left\{\mu_{\sigma}(x), \mu_{\sigma}(x \rightarrow y)\right\}, \\
& \gamma_{\sigma}(y) \leq \gamma_{\sigma}(x \odot(x \rightarrow y)) \leq \max \left\{\gamma_{\sigma}(x), \gamma_{\sigma}(x \rightarrow y)\right\}, \\
& \tilde{F}(y) \supseteq \tilde{F}(x \odot(x \rightarrow y)) \supseteq \tilde{F}(x) \cap \tilde{F}(x \rightarrow y)
\end{aligned}
$$

which proves (3.5).

Conversely, suppose that a soju structure $(A,\langle\sigma ; \tilde{F}\rangle)$ over $([0,1], U)$ satisfies (3.2) and (3.5). Let $x, y \in A$. Then $x \odot y \in A$ since $A$ is a sub-hoop of $E$. Since

$$
x \rightarrow(y \rightarrow(x \odot y))=(x \odot y) \rightarrow(x \odot y)=1 \in A,
$$

it follows from (3.2) and (3.5) that

$$
\begin{aligned}
\mu_{\sigma}(x \odot y) & \geq \min \left\{\mu_{\sigma}(y), \mu_{\sigma}(y \rightarrow(x \odot y))\right\} \\
& \geq \min \left\{\mu_{\sigma}(y), \min \left\{\mu_{\sigma}(x), \mu_{\sigma}(x \rightarrow(y \rightarrow(x \odot y)))\right\}\right\} \\
& =\min \left\{\mu_{\sigma}(y), \min \left\{\mu_{\sigma}(x), \mu_{\sigma}(1)\right\}\right\} \\
& =\min \left\{\mu_{\sigma}(y), \mu_{\sigma}(x)\right\},
\end{aligned}
$$




$$
\begin{aligned}
\gamma_{\sigma}(x \odot y) & \leq \max \left\{\gamma_{\sigma}(y), \gamma_{\sigma}(y \rightarrow(x \odot y))\right\} \\
& \leq \max \left\{\gamma_{\sigma}(y), \max \left\{\gamma_{\sigma}(x), \gamma_{\sigma}(x \rightarrow(y \rightarrow(x \odot y)))\right\}\right\} \\
& =\max \left\{\gamma_{\sigma}(y), \max \left\{\gamma_{\sigma}(x), \gamma_{\sigma}(1)\right\}\right\} \\
& =\max \left\{\gamma_{\sigma}(y), \gamma_{\sigma}(x)\right\},
\end{aligned}
$$

and

$$
\begin{aligned}
\tilde{F}(x \odot y) & \supseteq \tilde{F}(y) \cap \tilde{F}(y \rightarrow(x \odot y)) \\
& \supseteq \tilde{F}(y) \cap(\tilde{F}(x) \cap \tilde{F}(x \rightarrow(y \rightarrow(x \odot y)))) \\
& =\tilde{F}(y) \cap(\tilde{F}(x) \cap \tilde{F}(1)) \\
& =\tilde{F}(y) \cap \tilde{F}(x) .
\end{aligned}
$$

Let $x, y \in A$ be such that $x \leq y$. Then $x \rightarrow y=1 \in A$, and so

$$
\begin{aligned}
& \mu_{\sigma}(y) \geq \min \left\{\mu_{\sigma}(x), \mu_{\sigma}(x \rightarrow y)\right\}=\min \left\{\mu_{\sigma}(x), \mu_{\sigma}(1)\right\}=\mu_{\sigma}(x), \\
& \gamma_{\sigma}(y) \leq \max \left\{\gamma_{\sigma}(x), \gamma_{\sigma}(x \rightarrow y)\right\}=\max \left\{\gamma_{\sigma}(x), \gamma_{\sigma}(1)\right\}=\gamma_{\sigma}(x), \\
& \tilde{F}(y) \supseteq \tilde{F}(x) \cap \tilde{F}(x \rightarrow y)=\tilde{F}(x) \cap \tilde{F}(1)=\tilde{F}(x) .
\end{aligned}
$$

Therefore $(A,\langle\sigma ; \tilde{F}\rangle)$ is a soju $A$-filter of $E$.

Theorem 3.12. For any sub-hoop $A$ of a hoop algebra E, every soju $A$ filter is a soju A-sub-hoop.

PRoOF: Straightforward.

The following example shows that the converse of Theorem 3.12 is not true in general.

Example 3.13. Consider a hoop algebra $(E, \odot, \rightarrow, 1)$ in which $E=\{0, a, b, 1\}$ with binary operations $\rightarrow$ and $\odot$ which are given as follows:

\begin{tabular}{c|ccccc|cccc}
$\odot$ & 0 & $a$ & $b$ & 1 & $\rightarrow$ & 0 & $a$ & $b$ & 1 \\
\hline 0 & 0 & 0 & 0 & 0 & 0 & 1 & 1 & 1 & 1 \\
$a$ & 0 & $a$ & $a$ & $a$ & $a$ & 0 & 1 & 1 & 1 \\
$b$ & 0 & $a$ & $a$ & $b$ & $b$ & 0 & $b$ & 1 & 1 \\
1 & 0 & $a$ & $b$ & 1 & 1 & 0 & $a$ & $b$ & 1
\end{tabular}

Define a soju structure $(E,\langle\sigma ; \tilde{F}\rangle)$ over $([0,1], U)=([0,1], \mathbb{Z})$ by Table 4 . 
Table 4. Tabular representation of $(A,\langle\sigma ; \tilde{F}\rangle)$

\begin{tabular}{c||c|c}
\hline$A$ & $\sigma(x)=\left(\mu_{\sigma}(x), \gamma_{\sigma}(x)\right)$ & $\tilde{F}(x)$ \\
\hline 0 & $(0.65,0.30)$ & $8 \mathbb{N}$ \\
$a$ & $(0.45,0.25)$ & $4 \mathbb{Z}$ \\
$b$ & $(0.25,0.45)$ & $4 \mathbb{N}$ \\
1 & $(0.75,0.15)$ & $2 \mathbb{Z}$ \\
\hline
\end{tabular}

It is routine to verify that $(E,\langle\sigma ; \tilde{F}\rangle)$ is a soju sub-hoop of $E$. But it is not a soju filter of $E$ since $\gamma_{\sigma}(b)=0.45>0.25=\max \left\{\gamma_{\sigma}(a), \gamma_{\sigma}(a \rightarrow b)\right\}$.

Proposition 3.14. For any hoop algebra $E$, every soju $E$-filter $(E,\langle\sigma ; \tilde{F}\rangle)$ of $E$ satisfies:

$$
(\forall x, y \in E)\left(x \leq y \Rightarrow \mu_{\sigma}(x) \leq \mu_{\sigma}(y), \gamma_{\sigma}(x) \geq \gamma_{\sigma}(y), \tilde{F}(x) \subseteq \tilde{F}(y)\right)
$$

Proof: Let $x, y \in E$ be such that $x \leq y$. Then $x \rightarrow y=1$, and so

$$
\begin{aligned}
& \mu_{\sigma}(y) \geq \min \left\{\mu_{\sigma}(x), \mu_{\sigma}(x \rightarrow y)\right\}=\min \left\{\mu_{\sigma}(x), \mu_{\sigma}(1)\right\}=\mu_{\sigma}(x), \\
& \gamma_{\sigma}(y) \leq \max \left\{\gamma_{\sigma}(x), \gamma_{\sigma}(x \rightarrow y)\right\}=\max \left\{\gamma_{\sigma}(x), \gamma_{\sigma}(1)\right\}=\gamma_{\sigma}(x), \\
& \tilde{F}(y) \supseteq \tilde{F}(x) \cap \tilde{F}(x \rightarrow y)=\tilde{F}(x) \cap \tilde{F}(1)=\tilde{F}(x)
\end{aligned}
$$

by (3.2) and (3.5).

TheOREM 3.15. Given a hoop algebra $E$, the soju structure $(E,\langle\sigma ; \tilde{F}\rangle)$ over $([0,1], U)$ is a soju filter of $E$ if and only if its nonempty soju level sets $U\left(\mu_{\sigma} ; t\right), L\left(\gamma_{\sigma} ; s\right)$ and $i(\tilde{F} ; \alpha)$ are filters of $E$ for all $\alpha \in 2^{U}$ and $(t, s) \in[0,1] \times[0,1]$ with $t+s \leq 1$.

Proof: Assume that $(E,\langle\sigma ; \tilde{F}\rangle)$ is a soju filter of $E$ and let $\alpha \in 2^{U}$ and $(t, s) \in[0,1] \times[0,1]$ be such that $t+s \leq 1$, and $U\left(\mu_{\sigma} ; t\right), L\left(\gamma_{\sigma} ; s\right)$ and $i(\tilde{F} ; \alpha)$ are non-empty. It is clear that $1 \in U\left(\mu_{\sigma} ; t\right) \cap L\left(\gamma_{\sigma} ; s\right) \cap i(\tilde{F} ; \alpha)$. Let $x, y \in E$ be such that $x \in U\left(\mu_{\sigma} ; t\right) \cap L\left(\gamma_{\sigma} ; s\right) \cap i(\tilde{F} ; \alpha)$ and $x \rightarrow y \in$ $U\left(\mu_{\sigma} ; t\right) \cap L\left(\gamma_{\sigma} ; s\right) \cap i(\tilde{F} ; \alpha)$. Then $\mu_{\sigma}(x) \geq t, \gamma_{\sigma}(x) \leq s, \tilde{F}(x) \supseteq \alpha$, $\mu_{\sigma}(x \rightarrow y) \geq t, \gamma_{\sigma}(x \rightarrow y) \leq s, \tilde{F}(x \rightarrow y) \supseteq \alpha$. It follows from (3.5) that 


$$
\begin{aligned}
& \mu_{\sigma}(y) \geq \min \left\{\mu_{\sigma}(x), \mu_{\sigma}(x \rightarrow y)\right\} \geq t, \\
& \gamma_{\sigma}(y) \leq \max \left\{\gamma_{\sigma}(x), \gamma_{\sigma}(x \rightarrow y)\right\} \leq s, \\
& \tilde{F}(y) \supseteq \tilde{F}(x) \cap \tilde{F}(x \rightarrow y) \supseteq \alpha .
\end{aligned}
$$

Hence $y \in U\left(\mu_{\sigma} ; t\right) \cap L\left(\gamma_{\sigma} ; s\right) \cap i(\tilde{F} ; \alpha)$, and therefore $U\left(\mu_{\sigma} ; t\right), L\left(\gamma_{\sigma} ; s\right)$ and $i(\tilde{F} ; \alpha)$ are filters of $E$.

Conversely, suppose that the nonempty soju level sets $U\left(\mu_{\sigma} ; t\right), L\left(\gamma_{\sigma} ; s\right)$ and $i(\tilde{F} ; \alpha)$ of $(E,\langle\sigma ; \tilde{F}\rangle)$ are filters of $E$ for all $\alpha \in 2^{U}$ and $(t, s) \in$ $[0,1] \times[0,1]$ with $t+s \leq 1$. For any $x \in E$, let $\mu_{\sigma}(x)=t_{x}, \gamma_{\sigma}(x)=s_{x}$ and $\tilde{F}(x)=\alpha_{x}$. Then $x \in U\left(\mu_{\sigma} ; t_{x}\right) \cap L\left(\gamma_{\sigma} ; s_{x}\right) \cap i\left(\tilde{F} ; \alpha_{x}\right)$. Since $1 \in U\left(\mu_{\sigma} ; t_{x}\right) \cap$ $L\left(\gamma_{\sigma} ; s_{x}\right) \cap i\left(\tilde{F} ; \alpha_{x}\right)$, we have $\mu_{\sigma}(1) \geq t_{x}=\mu_{\sigma}(x), \gamma_{\sigma}(y) \leq s_{x}=\gamma_{\sigma}(x)$ and $\tilde{F}(y) \supseteq \alpha_{x}=\tilde{F}(x)$. For any $x, y \in E$, let $t_{x}, t_{y}, s_{x}, s_{y} \in[0,1]$ and $\alpha_{x}, \alpha_{y} \in 2^{U}$ be such that $\mu_{\sigma}(x)=t_{x}, \mu_{\sigma}(x \rightarrow y)=t_{y}, \gamma_{\sigma}(x)=s_{x}$, $\gamma_{\sigma}(x \rightarrow y)=s_{y}, \tilde{F}(x)=\alpha_{x}$ and $\tilde{F}(x \rightarrow y)=\alpha_{y}$. If we take $t:=$ $\min \left\{t_{x}, t_{x \rightarrow y}\right\}, s:=\max \left\{s_{x}, s_{x \rightarrow y}\right\}$ and $\alpha:=\alpha_{x} \cap \alpha_{x \rightarrow y}$, then $x, x \rightarrow y \in$ $U\left(\mu_{\sigma} ; t\right) \cap L\left(\gamma_{\sigma} ; s\right) \cap i(\tilde{F} ; \alpha)$. Thus $y \in U\left(\mu_{\sigma} ; t\right) \cap L\left(\gamma_{\sigma} ; s\right) \cap i(\tilde{F} ; \alpha)$, and so

$$
\begin{aligned}
& \mu_{\sigma}(y) \geq t=\min \left\{t_{x}, t_{y}\right\}=\min \left\{\mu_{\sigma}(x), \mu_{\sigma}(x \rightarrow y)\right\}, \\
& \gamma_{\sigma}(y) \leq s=\max \left\{s_{x}, s_{y}\right\}=\max \left\{\gamma_{\sigma}(x), \gamma_{\sigma}(x \rightarrow y)\right\}, \\
& \tilde{F}(y) \supseteq \alpha=\alpha_{x} \cap \alpha_{y}=\tilde{F}(x) \cap \tilde{F}(x \rightarrow y) .
\end{aligned}
$$

Therefore $(E,\langle\sigma ; \tilde{F}\rangle)$ is a soju filter of $E$.

TheOrem 3.16. For any sub-hoop A of a hoop algebra E, a soju structure $(A,\langle\sigma ; \tilde{F}\rangle)$ over $([0,1], U)$ is a soju A-filter of $E$ if and only if it satisfies (3.2) and

$$
(\forall x, y \in A)\left(\begin{array}{l}
\mu_{\sigma}(x \odot y)=\min \left\{\mu_{\sigma}(x), \mu_{\sigma}(y)\right\} \\
\gamma_{\sigma}(x \odot y)=\max \left\{\gamma_{\sigma}(x), \gamma_{\sigma}(y)\right\} \\
\tilde{F}(x \odot y)=\tilde{F}(x) \cap \tilde{F}(y)
\end{array}\right) .
$$

Proof: Assume that $(A,\langle\sigma ; \tilde{F}\rangle)$ is a soju $A$-filter of $E$ and let $x, y \in A$. Since $x \odot y \leq x$ and $x \odot y \leq y$, it follows from (3.4) that

$$
\begin{aligned}
& \mu_{\sigma}(x \odot y) \leq \min \left\{\mu_{\sigma}(x), \mu_{\sigma}(y)\right\}, \\
& \gamma_{\sigma}(x \odot y) \geq \max \left\{\gamma_{\sigma}(x), \gamma_{\sigma}(y)\right\}, \\
& \tilde{F}(x \odot y) \subseteq \tilde{F}(x) \cap \tilde{F}(y) .
\end{aligned}
$$


Since $x \leq y \rightarrow(x \odot y)$, we have

$$
\begin{aligned}
\mu_{\sigma}(x \odot y) & \geq \min \left\{\mu_{\sigma}(y), \mu_{\sigma}(y \rightarrow(x \odot y))\right\} \\
& \geq \min \left\{\mu_{\sigma}(x), \mu_{\sigma}(y)\right\} \\
\gamma_{\sigma}(x \odot y) & \leq \max \left\{\gamma_{\sigma}(y), \gamma_{\sigma}(y \rightarrow(x \odot y))\right\} \\
& \leq \max \left\{\gamma_{\sigma}(x), \gamma_{\sigma}(y)\right\}
\end{aligned}
$$

and

$$
\tilde{F}(x \odot y) \supseteq \tilde{F}(y) \cap \tilde{F}(y \rightarrow(x \odot y)) \supseteq \tilde{F}(x) \cap \tilde{F}(y)
$$

by (3.5) and (3.4). This proves (3.7).

Conversely, suppose that $(A,\langle\sigma ; \tilde{F}\rangle)$ satisfies (3.2) and (3.7). Since $x \odot(x \rightarrow y) \leq y$ for all $x, y \in A$, it follows from (3.2) and (3.7) that

$$
\begin{aligned}
& \mu_{\sigma}(y) \geq \mu_{\sigma}(x \odot(x \rightarrow y))=\min \left\{\mu_{\sigma}(x), \mu_{\sigma}(x \rightarrow y)\right\}, \\
& \gamma_{\sigma}(y) \leq \gamma_{\sigma}(x \odot(x \rightarrow y))=\max \left\{\gamma_{\sigma}(x), \gamma_{\sigma}(x \rightarrow y)\right\}, \\
& \tilde{F}(y) \supseteq \tilde{F}(x \odot(x \rightarrow y))=\tilde{F}(x) \cap \tilde{F}(x \rightarrow y) .
\end{aligned}
$$

Therefore $(A,\langle\sigma ; \tilde{F}\rangle)$ is a soju $A$-filter of $E$ by Theorem 3.11 .

TheOrem 3.17. For any sub-hoop A of a hoop algebra E, a soju structure $(A,\langle\sigma ; \tilde{F}\rangle)$ over $([0,1], U)$ is a soju $A$-filter of $E$ if and only if it satisfies (3.2) and

$$
(\forall x, y, z \in A)\left(\begin{array}{l}
\mu_{\sigma}(x \rightarrow z) \geq \min \left\{\mu_{\sigma}(x \rightarrow y), \mu_{\sigma}(y \rightarrow z)\right\} \\
\gamma_{\sigma}(x \rightarrow z) \leq \max \left\{\gamma_{\sigma}(x \rightarrow y), \gamma_{\sigma}(y \rightarrow z)\right\} \\
\tilde{F}(x \rightarrow z) \supseteq \tilde{F}(x \rightarrow y) \cap \tilde{F}(y \rightarrow z)
\end{array}\right) .
$$

Proof: Assume that $(A,\langle\sigma ; \tilde{F}\rangle)$ is a soju $A$-filter of $E$ and let $x, y, z \in A$. Since

$$
(x \rightarrow y) \odot(y \rightarrow z) \leq x \rightarrow z,
$$

we have

$$
\begin{aligned}
& \mu_{\sigma}(x \rightarrow z) \geq \mu_{\sigma}((x \rightarrow y) \odot(y \rightarrow z))=\min \left\{\mu_{\sigma}(x \rightarrow y), \mu_{\sigma}(y \rightarrow z)\right\}, \\
& \gamma_{\sigma}(x \rightarrow z) \leq \gamma_{\sigma}((x \rightarrow y) \odot(y \rightarrow z))=\max \left\{\gamma_{\sigma}(x \rightarrow y), \gamma_{\sigma}(y \rightarrow z)\right\}, \\
& \tilde{F}(x \rightarrow z) \supseteq \tilde{F}((x \rightarrow y) \odot(y \rightarrow z))=\tilde{F}(x \rightarrow y) \cap \tilde{F}(y \rightarrow z)
\end{aligned}
$$

by (3.4) and (3.7). 
Conversely, suppose that $(A,\langle\sigma ; \tilde{F}\rangle)$ satisfies (3.2) and (3.8). If we take $x=1$ in $(3.8)$, then we have $(3.5)$. Therefore $(A,\langle\sigma ; \tilde{F}\rangle)$ is a soju $A$-filter of $E$ by Theorem 3.11 .

THEOREM 3.18. For any sub-hoop A of a hoop algebra E, a soju structure $(A,\langle\sigma ; \tilde{F}\rangle)$ over $([0,1], U)$ is a soju A-filter of $E$ if and only if it satisfies (3.2) and

$$
(\forall x, y, z \in A)\left(\begin{array}{l}
\mu_{\sigma}(y \odot z) \geq \min \left\{\mu_{\sigma}(x \odot z), \mu_{\sigma}(x \rightarrow y)\right\} \\
\gamma_{\sigma}(y \odot z) \leq \max \left\{\gamma_{\sigma}(x \odot z), \gamma_{\sigma}(x \rightarrow y)\right\} \\
\tilde{F}(y \odot z) \supseteq \tilde{F}(x \odot z) \cap \tilde{F}(x \rightarrow y)
\end{array}\right) .
$$

Proof: Assume that $(A,\langle\sigma ; \tilde{F}\rangle)$ is a soju $A$-filter of $E$ and let $x, y, z \in A$. Note that $(z \odot x) \odot(x \rightarrow y)=z \odot(x \odot(x \rightarrow y)) \leq z \odot y$. Using (3.4) and (3.7), we get

$$
\begin{aligned}
& \mu_{\sigma}(z \odot y) \geq \mu_{\sigma}((z \odot x) \odot(x \rightarrow y))=\min \left\{\mu_{\sigma}(z \odot x), \mu_{\sigma}(x \rightarrow y)\right\}, \\
& \gamma_{\sigma}(z \odot y) \leq \gamma_{\sigma}((z \odot x) \odot(x \rightarrow y))=\max \left\{\gamma_{\sigma}(z \odot x), \gamma_{\sigma}(x \rightarrow y)\right\}, \\
& \tilde{F}(z \odot y) \supseteq \tilde{F}((z \odot x) \odot(x \rightarrow y))=\tilde{F}(z \odot x) \cap \tilde{F}(x \rightarrow y) .
\end{aligned}
$$

Conversely, suppose that $(A,\langle\sigma ; \tilde{F}\rangle)$ satisfies (3.2) and (3.9). If we take $z=1$ in $(3.9)$, then we have $(3.5)$. Therefore $(A,\langle\sigma ; \tilde{F}\rangle)$ is a soju $A$-filter of $E$ by Theorem 3.11 .

TheOrem 3.19. For any sub-hoop A of a hoop algebra E, a soju structure $(A,\langle\sigma ; \tilde{F}\rangle)$ over $([0,1], U)$ is a soju A-filter of $E$ if and only if it satisfies

$$
(\forall x, y, z \in A)\left(x \leq y \rightarrow z \Rightarrow\left\{\begin{array}{l}
\mu_{\sigma}(z) \geq \min \left\{\mu_{\sigma}(x), \mu_{\sigma}(y)\right\} \\
\gamma_{\sigma}(z) \leq \max \left\{\gamma_{\sigma}(x), \gamma_{\sigma}(y)\right\} \\
\tilde{F}(z) \supseteq \tilde{F}(x) \cap \tilde{F}(y)
\end{array}\right)\right.
$$

Proof: Assume that $(A,\langle\sigma ; \tilde{F}\rangle)$ is a soju $A$-filter of $E$ and let $x, y, z \in A$ be such that $x \leq y \rightarrow z$. Then $x \rightarrow(y \rightarrow z)=1$, and so

$$
\begin{aligned}
& \mu_{\sigma}(y \rightarrow z) \geq \min \left\{\mu_{\sigma}(x), \mu_{\sigma}(x \rightarrow(y \rightarrow z))\right\}=\min \left\{\mu_{\sigma}(x), \mu_{\sigma}(1)\right\}=\mu_{\sigma}(x), \\
& \gamma_{\sigma}(y \rightarrow z) \leq \max \left\{\gamma_{\sigma}(x), \gamma_{\sigma}(x \rightarrow(y \rightarrow z))\right\}=\max \left\{\gamma_{\sigma}(x), \gamma_{\sigma}(1)\right\}=\gamma_{\sigma}(x), \\
& \tilde{F}(y \rightarrow z) \supseteq \tilde{F}(x) \cap \tilde{F}(x \rightarrow(y \rightarrow z))=\tilde{F}(x) \cap \tilde{F}(1)=\tilde{F}(x)
\end{aligned}
$$


by (3.5). It follows that

$$
\begin{aligned}
& \mu_{\sigma}(z) \geq \min \left\{\mu_{\sigma}(y), \mu_{\sigma}(y \rightarrow z)\right\} \geq \min \left\{\mu_{\sigma}(x), \mu_{\sigma}(y)\right\}, \\
& \gamma_{\sigma}(z) \leq \max \left\{\gamma_{\sigma}(y), \gamma_{\sigma}(y \rightarrow z)\right\} \leq \max \left\{\gamma_{\sigma}(x), \gamma_{\sigma}(y)\right\}, \\
& \tilde{F}(z) \supseteq \tilde{F}(y) \cap \tilde{F}(y \rightarrow z)\} \supseteq \tilde{F}(x) \cap \tilde{F}(y) .
\end{aligned}
$$

Conversely, suppose that $(A,\langle\sigma ; \tilde{F}\rangle)$ satisfies (3.2) and (3.10). Since $x \leq(x \rightarrow y) \rightarrow y$ for all $x, y \in A$, it follows from (3.10) that

$$
\begin{aligned}
& \mu_{\sigma}(y) \geq \min \left\{\mu_{\sigma}(x), \mu_{\sigma}(x \rightarrow y)\right\}, \\
& \gamma_{\sigma}(y) \leq \max \left\{\gamma_{\sigma}(x), \gamma_{\sigma}(x \rightarrow y)\right\}, \\
& \tilde{F}(y) \supseteq \tilde{F}(x) \cap \tilde{F}(x \rightarrow y) .
\end{aligned}
$$

Hence $(A,\langle\sigma ; \tilde{F}\rangle)$ is a soju $A$-filter of $E$ by Theorem 3.11 .

TheOrem 3.20. For any sub-hoop A of a hoop algebra E, a soju structure $(A,\langle\sigma ; \tilde{F}\rangle)$ over $([0,1], U)$ is a soju A-filter of $E$ if and only if it satisfies (3.2) and

$$
(\forall x, y, z \in A)\left(\begin{array}{l}
\mu_{\sigma}(x \rightarrow z) \geq \min \left\{\mu_{\sigma}((x \rightarrow y) \rightarrow z), \mu_{\sigma}(y)\right\} \\
\gamma_{\sigma}(x \rightarrow z) \leq \max \left\{\gamma_{\sigma}((x \rightarrow y) \rightarrow z), \gamma_{\sigma}(y)\right\} \\
\tilde{F}(x \rightarrow z) \supseteq \tilde{F}((x \rightarrow y) \rightarrow z) \cap \tilde{F}(y)
\end{array}\right) .
$$

Proof: Suppose that $(A,\langle\sigma ; \tilde{F}\rangle)$ is a soju $A$-filter of $E$. Since $(x \rightarrow y) \rightarrow$ $z \leq y \rightarrow z$ and

$$
y \odot((x \rightarrow y) \rightarrow z) \leq y \odot(y \rightarrow z) \leq z \leq x \rightarrow z
$$

for all $x, y, z \in A$, we get

$$
\begin{aligned}
\mu_{\sigma}(x \rightarrow z) & \geq \mu_{\sigma}(z) \geq \mu_{\sigma}(y \odot(y \rightarrow z)) \\
& =\min \left\{\mu_{\sigma}(y), \mu_{\sigma}(y \rightarrow z)\right\} \\
& \geq \min \left\{\mu_{\sigma}(y), \mu_{\sigma}((x \rightarrow y) \rightarrow z)\right\} \\
\gamma_{\sigma}(x \rightarrow z) & \leq \gamma_{\sigma}(z) \leq \gamma_{\sigma}(y \odot(y \rightarrow z)) \\
& =\max \left\{\gamma_{\sigma}(y), \gamma_{\sigma}(y \rightarrow z)\right\} \\
& \leq \max \left\{\gamma_{\sigma}(y), \gamma_{\sigma}((x \rightarrow y) \rightarrow z)\right\}
\end{aligned}
$$


and

$$
\begin{aligned}
\tilde{F}(x \rightarrow z) & \supseteq \tilde{F}(z) \supseteq \tilde{F}(y \odot(y \rightarrow z)) \\
& =\tilde{F}(y) \cap \tilde{F}(y \rightarrow z) \\
& \supseteq \tilde{F}(y) \cap \tilde{F}((x \rightarrow y) \rightarrow z) .
\end{aligned}
$$

Conversely, assume that $(A,\langle\sigma ; \tilde{F}\rangle)$ satisfies (3.2) and (3.11). If we take $x=1$ in (3.11), then we have (3.5). Therefore $(A,\langle\sigma ; \tilde{F}\rangle)$ is a soju $A$-filter of $E$ by Theorem 3.11 .

Definition 3.21. Let $A$ be a subset of a hoop algebra $E$. A soju structure $(A,\langle\sigma ; \tilde{F}\rangle)$ over $([0,1], U)$ is called an implicative soju filter based on $A$ (briefly, implicative soju $A$-filter) of $E$ if it satisfies the condition (3.2) and

$$
(\forall x, y, z \in A)\left(\begin{array}{l}
\mu_{\sigma}(y) \geq \min \left\{\mu_{\sigma}(x), \mu_{\sigma}(x \rightarrow((y \rightarrow z) \rightarrow y))\right\} \\
\gamma_{\sigma}(y) \leq \max \left\{\gamma_{\sigma}(x), \gamma_{\sigma}(x \rightarrow((y \rightarrow z) \rightarrow y))\right\} \\
\tilde{F}(y) \supseteq \tilde{F}(x) \cap \tilde{F}(x \rightarrow((y \rightarrow z) \rightarrow y))
\end{array}\right) .
$$

Example 3.22. Consider a hoop $(H, \odot, \rightarrow, 1)$ in which $H=\{0, a, b, c, 1\}$ with binary operations $\odot$ and $\rightarrow$ which are given as follows:

\begin{tabular}{c|cccccc|ccccc}
$\odot$ & 0 & $a$ & $b$ & $c$ & 1 & $\rightarrow$ & 0 & $a$ & $b$ & $c$ & 1 \\
\hline 0 & 0 & 0 & 0 & 0 & 0 & 0 & 1 & 1 & 1 & 1 & 1 \\
$a$ & 0 & $a$ & $c$ & $c$ & $a$ & $a$ & 0 & 1 & $b$ & $b$ & 1 \\
$b$ & 0 & $c$ & $b$ & $c$ & $b$ & $b$ & 0 & $a$ & 1 & $a$ & 1 \\
$c$ & 0 & $c$ & $c$ & $c$ & $c$ & $c$ & 0 & 1 & 1 & 1 & 1 \\
1 & 0 & $a$ & $b$ & $c$ & 1 & 1 & 0 & $a$ & $b$ & $c$ & 1
\end{tabular}

Define a soju structure $(E,\langle\sigma ; \tilde{F}\rangle)$ over $([0,1], U)=([0,1], \mathbb{Z})$ by Table 5 . It is routine to check that $(E,\langle\sigma ; \tilde{F}\rangle)$ is an implicative soju filter of $E$.

Theorem 3.23. Given a sub-hoop A of a hoop algebra E, every implicative soju A-filter is a soju A-filter.

Proof: Let $(A,\langle\sigma ; \tilde{F}\rangle)$ be an implicative soju $A$-filter of $E$. If we take $z=1$ in $(3.12)$ and use $(a 5)$, then we have (3.5). Therefore $(A,\langle\sigma ; \tilde{F}\rangle)$ is a soju $A$-filter of $E$ by Theorem 3.11 .

The converse of Theorem 3.23 is not true in general as seen in the following example. 
Table 5. Tabular representation of $(E,\langle\sigma ; \tilde{F}\rangle)$

\begin{tabular}{c||c|c}
\hline$E$ & $\sigma(x)=\left(\mu_{\sigma}(x), \gamma_{\sigma}(x)\right)$ & $\tilde{F}(x)$ \\
\hline 0 & $(0.3,0.6)$ & $3 \mathbb{N}$ \\
$a$ & $(0.6,0.2)$ & $3 \mathbb{Z}$ \\
$b$ & $(0.6,0.2)$ & $3 \mathbb{Z}$ \\
$c$ & $(0.6,0.2)$ & $3 \mathbb{Z}$ \\
1 & $(0.6,0.2)$ & $3 \mathbb{Z}$ \\
\hline
\end{tabular}

Example 3.24. Consider the hoop algebra $E$ in Example 3.22 and let $(E$, $\langle\sigma ; \tilde{F}\rangle)$ be a soju structure over $([0,1], U)=([0,1], \mathbb{Z})$ defined by Table 6 .

Table 6. Tabular representation of $(E,\langle\sigma ; \tilde{F}\rangle)$

\begin{tabular}{c||c|c}
\hline$E$ & $\sigma(x)=\left(\mu_{\sigma}(x), \gamma_{\sigma}(x)\right)$ & $\tilde{F}(x)$ \\
\hline 0 & $(0.1,0.7)$ & $8 \mathbb{N}$ \\
$a$ & $(0.6,0.3)$ & $4 \mathbb{Z}$ \\
$b$ & $(0.3,0.5)$ & $4 \mathbb{N}$ \\
$c$ & $(0.3,0.5)$ & $4 \mathbb{N}$ \\
1 & $(0.7,0.2)$ & $2 \mathbb{Z}$ \\
\hline
\end{tabular}

It is routine to check that $(E,\langle\sigma ; \tilde{F}\rangle)$ is a soju filter of $E$. But it is not an implicative soju filter of $E$ since $\mu_{\sigma}(b)=0.3<0.6=\min \left\{\mu_{\sigma}(a), \mu_{\sigma}(a \rightarrow\right.$ $((b \rightarrow 0) \rightarrow b))\}$.

Proposition 3.25. Given a sub-hoop $A$ of a hoop algebra $E$, every implicative soju $A$-filter $(A,\langle\sigma ; \tilde{F}\rangle)$ of $E$ satisfies the following assertions.

$$
\begin{aligned}
& (\forall x, y \in A)\left(\begin{array}{l}
\mu_{\sigma}((x \rightarrow y) \rightarrow x) \leq \mu_{\sigma}(x) \\
\gamma_{\sigma}((x \rightarrow y) \rightarrow x) \geq \gamma_{\sigma}(x) \\
\tilde{F}((x \rightarrow y) \rightarrow x) \subseteq \tilde{F}(x)
\end{array}\right) . \\
& (\forall x, y \in A)\left(\begin{array}{l}
\mu_{\sigma}(((x \rightarrow y) \rightarrow x) \rightarrow x)=\mu_{\sigma}(1) \\
\gamma_{\sigma}(((x \rightarrow y) \rightarrow x) \rightarrow x)=\gamma_{\sigma}(1) \\
\tilde{F}(((x \rightarrow y) \rightarrow x) \rightarrow x)=\tilde{F}(1)
\end{array}\right) .
\end{aligned}
$$


Proof: Let $A$ be a sub-hoop and $(A,\langle\sigma ; \tilde{F}\rangle)$ an implicative soju $A$-filter of a hoop algebra $E$. If we put $y=x, x=1$ and $z=y$ in (3.12) and use (a5) and (3.2), then we have (3.13). Using (3.13), (H1), (a5), (a7), (a9) and (3.4), we have

$$
\begin{aligned}
\mu_{\sigma}(((x \rightarrow y) \rightarrow x) \rightarrow x) & \geq \mu_{\sigma}(((((x \rightarrow y) \rightarrow x) \rightarrow x) \rightarrow y) \rightarrow(((x \rightarrow y) \rightarrow x) \rightarrow x)) \\
& \left.=\mu_{\sigma}(((x \rightarrow y) \rightarrow x) \rightarrow((((x \rightarrow y) \rightarrow x) \rightarrow x) \rightarrow y) \rightarrow x)\right) \\
& \geq \mu_{\sigma}(((((x \rightarrow y) \rightarrow x) \rightarrow x) \rightarrow y) \rightarrow(x \rightarrow y)) \\
& \geq \mu_{\sigma}(x \rightarrow(((x \rightarrow y) \rightarrow x) \rightarrow x)) \\
& =\mu_{\sigma}(((x \rightarrow y) \rightarrow x) \rightarrow(x \rightarrow x)) \\
& =\mu_{\sigma}(((x \rightarrow y) \rightarrow x) \rightarrow 1) \\
& =\mu_{\sigma}(1),
\end{aligned}
$$

$$
\begin{aligned}
\gamma_{\sigma}(((x \rightarrow y) \rightarrow x) \rightarrow x) & \leq \gamma_{\sigma}(((((x \rightarrow y) \rightarrow x) \rightarrow x) \rightarrow y) \rightarrow(((x \rightarrow y) \rightarrow x) \rightarrow x)) \\
& \left.=\gamma_{\sigma}(((x \rightarrow y) \rightarrow x) \rightarrow((((x \rightarrow y) \rightarrow x) \rightarrow x) \rightarrow y) \rightarrow x)\right) \\
& \leq \gamma_{\sigma}(((((x \rightarrow y) \rightarrow x) \rightarrow x) \rightarrow y) \rightarrow(x \rightarrow y)) \\
& \leq \gamma_{\sigma}(x \rightarrow(((x \rightarrow y) \rightarrow x) \rightarrow x)) \\
& =\gamma_{\sigma}(((x \rightarrow y) \rightarrow x) \rightarrow(x \rightarrow x)) \\
& =\gamma_{\sigma}(((x \rightarrow y) \rightarrow x) \rightarrow 1) \\
& =\gamma_{\sigma}(1),
\end{aligned}
$$

$$
\begin{aligned}
\tilde{F}(((x \rightarrow y) \rightarrow x) \rightarrow x) & \supseteq \tilde{F}(((((x \rightarrow y) \rightarrow x) \rightarrow x) \rightarrow y) \rightarrow(((x \rightarrow y) \rightarrow x) \rightarrow x)) \\
& =\tilde{F}(((x \rightarrow y) \rightarrow x) \rightarrow((((x \rightarrow y) \rightarrow x) \rightarrow x) \rightarrow y) \rightarrow x)) \\
& \supseteq \tilde{F}(((((x \rightarrow y) \rightarrow x) \rightarrow x) \rightarrow y) \rightarrow(x \rightarrow y)) \\
& \supseteq \tilde{F}(x \rightarrow(((x \rightarrow y) \rightarrow x) \rightarrow x)) \\
& =\tilde{F}(((x \rightarrow y) \rightarrow x) \rightarrow(x \rightarrow x)) \\
& =\tilde{F}(((x \rightarrow y) \rightarrow x) \rightarrow 1) \\
& =\tilde{F}(1)
\end{aligned}
$$

for all $x, y \in A$. It follows from (3.2) that we have (3.14). 
Proposition 3.26. Given a sub-hoop $A$ of a bounded hoop algebra $E$, every implicative soju $A$-filter $(A,\langle\sigma ; \tilde{F}\rangle)$ of $E$ satisfies the following assertions.

$$
\begin{aligned}
& (\forall x \in A)\left(\begin{array}{l}
\mu_{\sigma}\left(\left(x^{\prime} \rightarrow x\right) \rightarrow x\right)=\mu_{\sigma}(1) \\
\gamma_{\sigma}\left(\left(x^{\prime} \rightarrow x\right) \rightarrow x\right)=\gamma_{\sigma}(1) \\
\tilde{F}\left(\left(x^{\prime} \rightarrow x\right) \rightarrow x\right)=\tilde{F}(1)
\end{array}\right) \\
& (\forall x, y \in A)\left(\begin{array}{l}
\mu_{\sigma}(x \rightarrow y) \geq \mu_{\sigma}\left(\left(x \odot y^{\prime}\right) \rightarrow y\right) \\
\gamma_{\sigma}(x \rightarrow y) \leq \gamma_{\sigma}\left(\left(x \odot y^{\prime}\right) \rightarrow y\right) \\
\tilde{F}(x \rightarrow y) \supseteq \tilde{F}\left(\left(x \odot y^{\prime}\right) \rightarrow y\right)
\end{array}\right) \\
& (\forall x, y, z \in A)\left(\begin{array}{l}
\mu_{\sigma}(x \rightarrow z) \geq \min \left\{\mu_{\sigma}(y \rightarrow z), \mu_{\sigma}\left(x \rightarrow\left(z^{\prime} \rightarrow y\right)\right)\right\} \\
\gamma_{\sigma}(x \rightarrow z) \leq \max \left\{\gamma_{\sigma}(y \rightarrow z), \gamma_{\sigma}\left(x \rightarrow\left(z^{\prime} \rightarrow y\right)\right)\right\} \\
\tilde{F}(x \rightarrow z) \supseteq \tilde{F}(y \rightarrow z) \cap \tilde{F}\left(x \rightarrow\left(z^{\prime} \rightarrow y\right)\right)
\end{array}\right) .
\end{aligned}
$$

Proof: Let $A$ be a sub-hoop and $(A,\langle\sigma ; \tilde{F}\rangle)$ an implicative soju $A$-filter of a bounded hoop algebra $E$. Then $(A,\langle\sigma ; F\rangle)$ is a soju $A$-filter of $E$ (see Theorem 3.23). If we take $y=0$ in (3.14), then

$$
\begin{aligned}
& \mu_{\sigma}\left(\left(x^{\prime} \rightarrow x\right) \rightarrow x\right)=\mu_{\sigma}(((x \rightarrow 0) \rightarrow x) \rightarrow x)=\mu_{\sigma}(1), \\
& \gamma_{\sigma}\left(\left(x^{\prime} \rightarrow x\right) \rightarrow x\right)=\gamma_{\sigma}(((x \rightarrow 0) \rightarrow x) \rightarrow x)=\gamma_{\sigma}(1), \\
& \tilde{F}\left(\left(x^{\prime} \rightarrow x\right) \rightarrow x\right)=\tilde{F}(((x \rightarrow 0) \rightarrow x) \rightarrow x)=\tilde{F}(1)
\end{aligned}
$$

for all $x \in E$. Note that $y^{\prime} \rightarrow(x \rightarrow y) \leq(x \rightarrow y)^{\prime} \rightarrow(x \rightarrow y)$ for all $x, y \in E$. It follows from (H3) and (3.6) that

$$
\begin{aligned}
& \mu_{\sigma}\left(\left(x \odot y^{\prime}\right) \rightarrow y\right)=\mu_{\sigma}\left(y^{\prime} \rightarrow(x \rightarrow y)\right) \leq \mu_{\sigma}\left((x \rightarrow y)^{\prime} \rightarrow(x \rightarrow y)\right), \\
& \gamma_{\sigma}\left(\left(x \odot y^{\prime}\right) \rightarrow y\right)=\gamma_{\sigma}\left(y^{\prime} \rightarrow(x \rightarrow y)\right) \geq \gamma_{\sigma}\left((x \rightarrow y)^{\prime} \rightarrow(x \rightarrow y)\right), \\
& \tilde{F}\left(\left(x \odot y^{\prime}\right) \rightarrow y\right)=\tilde{F}\left(y^{\prime} \rightarrow(x \rightarrow y)\right) \subseteq \tilde{F}\left((x \rightarrow y)^{\prime} \rightarrow(x \rightarrow y)\right) .
\end{aligned}
$$

Combining (a5), (3.2), (3.12) and (3.18) induce 


$$
\begin{aligned}
\mu_{\sigma}(x \rightarrow y) & \geq \min \left\{\mu_{\sigma}(1), \mu_{\sigma}(1 \rightarrow(((x \rightarrow y) \rightarrow 0) \rightarrow(x \rightarrow y)))\right\} \\
& =\mu_{\sigma}(((x \rightarrow y) \rightarrow 0) \rightarrow(x \rightarrow y)) \\
& =\mu_{\sigma}\left((x \rightarrow y)^{\prime} \rightarrow(x \rightarrow y)\right) \\
& \geq \mu_{\sigma}\left(\left(x \odot y^{\prime}\right) \rightarrow y\right), \\
\gamma_{\sigma}(x \rightarrow y) & \leq \max \left\{\gamma_{\sigma}(1), \gamma_{\sigma}(1 \rightarrow(((x \rightarrow y) \rightarrow 0) \rightarrow(x \rightarrow y)))\right\} \\
& =\gamma_{\sigma}(((x \rightarrow y) \rightarrow 0) \rightarrow(x \rightarrow y)) \\
& =\gamma_{\sigma}\left((x \rightarrow y)^{\prime} \rightarrow(x \rightarrow y)\right) \\
& \leq \gamma_{\sigma}\left(\left(x \odot y^{\prime}\right) \rightarrow y\right),
\end{aligned}
$$

and

$$
\begin{aligned}
\tilde{F}(x \rightarrow y) & \supseteq \mu_{\sigma}(1) \cap \mu_{\sigma}(1 \rightarrow(((x \rightarrow y) \rightarrow 0) \rightarrow(x \rightarrow y))) \\
& =\tilde{F}(((x \rightarrow y) \rightarrow 0) \rightarrow(x \rightarrow y)) \\
& =\tilde{F}\left((x \rightarrow y)^{\prime} \rightarrow(x \rightarrow y)\right) \\
& \supseteq \tilde{F}\left(\left(x \odot y^{\prime}\right) \rightarrow y\right) .
\end{aligned}
$$

This proves (3.16). Using (HP3), (3.8) and (3.16) impliy that

$$
\begin{aligned}
\mu_{\sigma}(x \rightarrow z) & \geq \mu_{\sigma}\left(\left(x \odot z^{\prime}\right) \rightarrow z\right) \geq \min \left\{\mu_{\sigma}\left(\left(x \odot z^{\prime}\right) \rightarrow y\right), \mu_{\sigma}(y \rightarrow z)\right\} \\
& =\min \left\{\mu_{\sigma}\left(x \rightarrow\left(z^{\prime} \rightarrow y\right)\right), \mu_{\sigma}(y \rightarrow z)\right\}, \\
\gamma_{\sigma}(x \rightarrow z) & \leq \gamma_{\sigma}\left(\left(x \odot z^{\prime}\right) \rightarrow z\right) \leq \max \left\{\gamma_{\sigma}\left(\left(x \odot z^{\prime}\right) \rightarrow y\right), \gamma_{\sigma}(y \rightarrow z)\right\} \\
& =\max \left\{\gamma_{\sigma}\left(x \rightarrow\left(z^{\prime} \rightarrow y\right)\right), \gamma_{\sigma}(y \rightarrow z)\right\},
\end{aligned}
$$

and

$$
\begin{aligned}
\tilde{F}(x \rightarrow z) & \supseteq \mu_{\sigma}\left(\left(x \odot z^{\prime}\right) \rightarrow z\right) \supseteq \tilde{F}\left(\left(x \odot z^{\prime}\right) \rightarrow y\right) \cap \tilde{F}(y \rightarrow z) \\
& =\tilde{F}\left(x \rightarrow\left(z^{\prime} \rightarrow y\right)\right) \cap \tilde{F}(y \rightarrow z),
\end{aligned}
$$

which proves (3.17).

TheOrem 3.27. Given a hoop algebra $E$, the soju structure $(E,\langle\sigma ; \tilde{F}\rangle)$ over $([0,1], U)$ is an implicative soju filter of $E$ if and only if its nonempty soju level sets $U\left(\mu_{\sigma} ; t\right), L\left(\gamma_{\sigma} ; s\right)$ and $i(\tilde{F} ; \alpha)$ are implicative filters of $E$ for all $\alpha \in 2^{U}$ and $(t, s) \in[0,1] \times[0,1]$ with $t+s \leq 1$.

Proof: It is similar to the proof of Theorem 3.15. 
We provide conditions for a soju $A$-filter to be an implicative soju $A$-filter.

Theorem 3.28. Let $A$ be a sub-hoop of a hoop algebra E. If a soju A-filter $(A,\langle\sigma ; \tilde{F}\rangle)$ of $E$ satisfies the condition (3.14), then it is an implicative soju A-filter of $E$.

Proof: Let $x, y, z \in A$. Then

$$
\begin{aligned}
\mu_{\sigma}(y) & \geq \min \left\{\mu_{\sigma}(((y \rightarrow z) \rightarrow y) \rightarrow y), \mu_{\sigma}((y \rightarrow z) \rightarrow y)\right\} \\
& =\min \left\{\mu_{\sigma}(1), \mu_{\sigma}((y \rightarrow z) \rightarrow y)\right\}=\mu_{\sigma}((y \rightarrow z) \rightarrow y) \\
& \geq \min \left\{\mu_{\sigma}(x), \mu_{\sigma}(x \rightarrow((y \rightarrow z) \rightarrow y))\right\}, \\
\gamma_{\sigma}(y) & \leq \max \left\{\gamma_{\sigma}(((y \rightarrow z) \rightarrow y) \rightarrow y), \gamma_{\sigma}((y \rightarrow z) \rightarrow y)\right\} \\
& =\max \left\{\gamma_{\sigma}(1), \gamma_{\sigma}((y \rightarrow z) \rightarrow y)\right\}=\gamma_{\sigma}((y \rightarrow z) \rightarrow y) \\
& \leq \max \left\{\gamma_{\sigma}(x), \gamma_{\sigma}(x \rightarrow((y \rightarrow z) \rightarrow y))\right\},
\end{aligned}
$$

and

$$
\begin{aligned}
\tilde{F}(y) & \supseteq \tilde{F}(((y \rightarrow z) \rightarrow y) \rightarrow y) \cap \tilde{F}((y \rightarrow z) \rightarrow y) \\
& =\tilde{F}(1) \cap \tilde{F}((y \rightarrow z) \rightarrow y)=\tilde{F}((y \rightarrow z) \rightarrow y) \\
& \supseteq \tilde{F}(x) \cap \tilde{F}(x \rightarrow((y \rightarrow z) \rightarrow y))
\end{aligned}
$$

by (3.2), (3.5) and (3.14). Therefore $(A,\langle\sigma ; \tilde{F}\rangle)$ is an implicative soju $A$-filter of $E$.

Theorem 3.29. Let $A$ be a sub-hoop of a hoop algebra E. If a soju A-filter $(A,\langle\sigma ; \tilde{F}\rangle)$ of $E$ satisfies the condition (3.13), then it is an implicative soju $A$-filter of $E$.

Proof: Let $(A,\langle\sigma ; \tilde{F}\rangle)$ be a soju $A$-filter of $E$ which satisfies (3.13). We have shown that (3.13) implies (3.14) in Proposition 3.25. Therefore $(A$, $\langle\sigma ; \tilde{F}\rangle)$ is an implicative soju $A$-filter of $E$ by Theorem 3.28.

THEOREM 3.30. Let $A$ be a sub-hoop of a hoop algebra E. If a soju A-filter $(A,\langle\sigma ; \tilde{F}\rangle)$ of $E$ satisfies the condition (3.15), then it is an implicative soju $A$-filter of $E$.

Proof: Let $(A,\langle\sigma ; \tilde{F}\rangle)$ be a soju $A$-filter of $E$ which satisfies (3.15). Note that 


$$
\left(x^{\prime} \rightarrow x\right) \rightarrow x \leq((x \rightarrow y) \rightarrow x) \rightarrow x
$$

for all $x, y \in E$. It follows from (3.2), (3.6) and (3.15) that

$$
\begin{aligned}
& \mu_{\sigma}(((x \rightarrow y) \rightarrow x) \rightarrow x) \leq \mu_{\sigma}(1)=\mu_{\sigma}\left(\left(x^{\prime} \rightarrow x\right) \rightarrow x\right) \leq \mu_{\sigma}(((x \rightarrow y) \rightarrow x) \rightarrow x), \\
& \gamma_{\sigma}(((x \rightarrow y) \rightarrow x) \rightarrow x) \geq \gamma_{\sigma}(1)=\gamma_{\sigma}\left(\left(x^{\prime} \rightarrow x\right) \rightarrow x\right) \geq \gamma_{\sigma}(((x \rightarrow y) \rightarrow x) \rightarrow x), \\
& \tilde{F}(((x \rightarrow y) \rightarrow x) \rightarrow x) \subseteq \tilde{F}(1)=\tilde{F}\left(\left(x^{\prime} \rightarrow x\right) \rightarrow x\right) \subseteq \tilde{F}(((x \rightarrow y) \rightarrow x) \rightarrow x) .
\end{aligned}
$$

Thus (3.14) is valid, and therefore $(A,\langle\sigma ; \tilde{F}\rangle)$ is an implicative soju $A$-filter of $E$ by Theorem 3.28.

Theorem 3.31. Let $A$ be a sub-hoop of a hoop algebra E. If a soju A-filter $(A,\langle\sigma ; \tilde{F}\rangle)$ of $E$ satisfies the condition (3.16), then it is an implicative soju $A$-filter of $E$.

Proof: Let $(A,\langle\sigma ; \tilde{F}\rangle)$ be a soju $A$-filter of $E$ which satisfies (3.16). For any $x, y \in A$, we have

$$
\begin{aligned}
\mu_{\sigma}\left(\left(x^{\prime} \rightarrow x\right) \rightarrow x\right) & \leq \mu_{\sigma}(1)=\mu_{\sigma}\left(\left(x^{\prime} \rightarrow x\right) \rightarrow\left(x^{\prime} \rightarrow x\right)\right) \\
& =\mu_{\sigma}\left(\left(x^{\prime} \odot\left(x^{\prime} \rightarrow x\right)\right) \rightarrow x\right) \leq \mu_{\sigma}\left(\left(x^{\prime} \rightarrow x\right) \rightarrow x\right), \\
\gamma_{\sigma}\left(\left(x^{\prime} \rightarrow x\right) \rightarrow x\right) & \geq \gamma_{\sigma}(1)=\gamma_{\sigma}\left(\left(x^{\prime} \rightarrow x\right) \rightarrow\left(x^{\prime} \rightarrow x\right)\right) \\
& =\gamma_{\sigma}\left(\left(x^{\prime} \odot\left(x^{\prime} \rightarrow x\right)\right) \rightarrow x\right) \geq \gamma_{\sigma}\left(\left(x^{\prime} \rightarrow x\right) \rightarrow x\right),
\end{aligned}
$$

and

$$
\begin{aligned}
\tilde{F}\left(\left(x^{\prime} \rightarrow x\right) \rightarrow x\right) & \subseteq \tilde{F}(1)=\tilde{F}\left(\left(x^{\prime} \rightarrow x\right) \rightarrow\left(x^{\prime} \rightarrow x\right)\right) \\
& =\tilde{F}\left(\left(x^{\prime} \odot\left(x^{\prime} \rightarrow x\right)\right) \rightarrow x\right) \subseteq \tilde{F}\left(\left(x^{\prime} \rightarrow x\right) \rightarrow x\right)
\end{aligned}
$$

by (H1), (H3), (3.2) and (3.16). Thus (3.15) is valid, and therefore ( $A$, $\langle\sigma ; \tilde{F}\rangle)$ is an implicative soju $A$-filter of $E$ by Theorem 3.30 .

Theorem 3.32. Let $A$ be a sub-hoop of a hoop algebra E. If a soju A-filter $(A,\langle\sigma ; \tilde{F}\rangle)$ of $E$ satisfies the condition (3.17), then it is an implicative soju $A$-filter of $E$.

Proof: Let $(A,\langle\sigma ; \tilde{F}\rangle)$ be a soju $A$-filter of $E$ which satisfies (3.17). The condition (3.17) implies that 


$$
\begin{aligned}
\mu_{\sigma}(x \rightarrow y) & \geq \min \left\{\mu_{\sigma}(y \rightarrow y), \mu_{\sigma}\left(x \rightarrow\left(y^{\prime} \rightarrow y\right)\right)\right\} \\
& =\min \left\{\mu_{\sigma}(1), \mu_{\sigma}\left(\left(x \odot y^{\prime}\right) \rightarrow y\right)\right\} \\
& =\mu_{\sigma}\left(\left(x \odot y^{\prime}\right) \rightarrow y\right), \\
\gamma_{\sigma}(x \rightarrow y) & \leq \max \left\{\gamma_{\sigma}(y \rightarrow y), \gamma_{\sigma}\left(x \rightarrow\left(y^{\prime} \rightarrow y\right)\right)\right\} \\
& =\max \left\{\gamma_{\sigma}(1), \gamma_{\sigma}\left(\left(x \odot y^{\prime}\right) \rightarrow y\right)\right\} \\
& =\gamma_{\sigma}\left(\left(x \odot y^{\prime}\right) \rightarrow y\right),
\end{aligned}
$$

and

$$
\begin{aligned}
\tilde{F}(x \rightarrow y) & \supseteq \tilde{F}(y \rightarrow y) \cap \tilde{F}\left(x \rightarrow\left(y^{\prime} \rightarrow y\right)\right) \\
& =\tilde{F}(1) \cap \tilde{F}\left(\left(x \odot y^{\prime}\right) \rightarrow y\right) \\
& =\tilde{F}\left(\left(x \odot y^{\prime}\right) \rightarrow y\right)
\end{aligned}
$$

for all $x, y \in A$. Hence (3.16) is valid, and therefore $(A,\langle\sigma ; \tilde{F}\rangle)$ is an implicative soju $A$-filter of $E$ by Theorem 3.31 .

TheOrem 3.33. (Extension property) Let $(E,\langle\sigma ; \tilde{F}\rangle)$ and $(E,\langle\rho ; \tilde{G}\rangle)$ be soju filters of a hoop algebra $E$ such that

$$
\mu_{\sigma}(1)=\mu_{\rho}(1), \gamma_{\sigma}(1)=\gamma_{\rho}(1), \tilde{F}(1)=\tilde{G}(1)
$$

and $(E,\langle\sigma ; \tilde{F}\rangle) \Subset(E,\langle\rho ; \tilde{G}\rangle)$, that is,

$$
(\forall x \in E)\left(\mu_{\sigma}(x) \leq \mu_{\rho}(x), \gamma_{\sigma}(x) \geq \gamma_{\rho}(x), \tilde{F}(x) \subseteq \tilde{G}(x)\right) .
$$

If $(E,\langle\sigma ; \tilde{F}\rangle)$ is an impliative soju filter of $E$, then so is $(E,\langle\rho ; \tilde{G}\rangle)$.

Proof: Assume that $(E,\langle\sigma ; \tilde{F}\rangle)$ is an impliative soju filter of $E$. Then $(E,\langle\sigma ; \tilde{F}\rangle)$ is a soju filter of $E$ (see Theorem 3.23). For any $x, y \in E$, we have

$$
\begin{aligned}
& \mu_{\rho}\left(\left(x^{\prime} \rightarrow x\right) \rightarrow x\right) \geq \mu_{\sigma}\left(\left(x^{\prime} \rightarrow x\right) \rightarrow x\right)=\mu_{\sigma}(1)=\mu_{\rho}(1), \\
& \gamma_{\rho}\left(\left(x^{\prime} \rightarrow x\right) \rightarrow x\right) \leq \gamma_{\sigma}\left(\left(x^{\prime} \rightarrow x\right) \rightarrow x\right)=\gamma_{\sigma}(1)=\gamma_{\rho}(1), \\
& \tilde{G}\left(\left(x^{\prime} \rightarrow x\right) \rightarrow x\right) \supseteq \tilde{F}\left(\left(x^{\prime} \rightarrow x\right) \rightarrow x\right)=\tilde{F}(1)=\tilde{G}(1)
\end{aligned}
$$


by $(3.19),(3.20)$ and $(3.15)$. Since $(E,\langle\sigma ; \tilde{F}\rangle)$ is a soju filter of $E$, it follows from (3.2) that $\mu_{\rho}\left(\left(x^{\prime} \rightarrow x\right) \rightarrow x\right) \leq \mu_{\rho}(1), \gamma_{\rho}\left(\left(x^{\prime} \rightarrow x\right) \rightarrow x\right) \geq \gamma_{\rho}(1)$ and $\tilde{G}\left(\left(x^{\prime} \rightarrow x\right) \rightarrow x\right) \subseteq \tilde{G}(1)$. Hence $\mu_{\rho}\left(\left(x^{\prime} \rightarrow x\right) \rightarrow x\right)=\mu_{\rho}(1), \gamma_{\rho}\left(\left(x^{\prime} \rightarrow\right.\right.$ $x) \rightarrow x)=\gamma_{\rho}(1)$ and $\tilde{G}\left(\left(x^{\prime} \rightarrow x\right) \rightarrow x\right)=\tilde{G}(1)$ for all $x, y \in E$. Therefore $(E,\langle\sigma ; \tilde{F}\rangle)$ is an implicative soju filter of $E$ by Theorem 3.30 .

\section{References}

[1] K. T. Atanassov, Intuitionistic fuzzy sets, Fuzzy Sets and Systems, vol. 20(1) (1986), pp. 87-96, DOI: https://doi.org/10.1016/S0165-0114(86) 80034-3.

[2] K. T. Atanassov, New operations defined over the intuitionistic fuzzy sets, Fuzzy Sets and Systems, vol. 61(2) (1994), pp. 137-142, DOI: https: //doi.org/10.1016/0165-0114(94)90229-1.

[3] W. J. Blok, I. M. A. Ferreirim, Hoops and their implicational reducts, Logical Computer Sciences, vol. 28 (1993), pp. 219-230.

[4] W. J. Blok, I. M. A. Ferreirim, On the structure of hoops, Algebra Universalis, vol. 43 (2000), pp. 233-257, DOI: https://doi.org/10.1007/ s000120050156.

[5] R. A. Borzooei, M. A. Kologani, Local and perfect semihoops, Journal of Intelligent and Fuzzy Systems, vol. 29(1) (2015), pp. 223-234, DOI: https://doi.org/10.3233/IFS-151589.

[6] R. A. Borzooei, M. A. Kologani, M. S. Kish, Y. B. Jun, Fuzzy positive implicative filters of hoops based on fuzzy points, Mathematics, vol. 7 (2019), p. 566, DOI: https://doi.org/10.3390/math7060566.

[7] R. A. Borzooei, H. R. Varasteh, K. Borna, Fundamental hoop-algebras, Ratio Mathematica, vol. 29 (2015), pp. 25-40.

[8] B. Bosbach, Komplementäre Halbgruppen. Axiomatik und Arithmetik, Fundamenta Mathematicae, vol. 64(3) (1969), pp. 257-287, DOI: https: //doi.org/10.4064/FM-64-3-257-287.

[9] B. Bosbach, Komplementäre Halbgruppen. Kongruenzen und Quotienten, Fundamenta Mathematicae, vol. 69(1) (1970), pp. 1-14.

[10] G. Georgescu, L. Leustean, V. Preoteasa, Pseudo-hoops, Journal of Multiple-Valued Logic and Soft Computing, vol. 11(1-2) (2005), pp. 153-184. 
[11] Y. B. Jun, S. Z. Song, E. H. Roh, Soju structures with applications in $B C K / B C I$-algebras, Afrika Matematika, (submitted).

[12] M. A. Kologani, R. A. Borzooei, On ideal theory of hoops, Mathematica Bohemica, vol. 145 (2019), pp. 1-22, DOI: https://doi.org/10.21136/MB. 2019.0140-17.

[13] M. Kondo, Some types of filters in hoops, Multiple-Valued Logic, (ISMVL), (2011), pp. 50-53, DOI: https://doi.org/10.1109/ISMVL.2011.9.

[14] P. K. Maji, R. Biswas, A. R. Roy, Soft set theory, Computers and Mathematics with Applications, vol. 45(4-5) (2003), pp. 555-562, DOI: https://doi.org/10.1016/S0898-1221(03)00016-6.

[15] D. Molodtsov, Soft set theory - First results, Computers and Mathematics with Applications, vol. 37(4-5) (1999), pp. 19-31, DOI: https://doi.org/10.1016/S0898-1221(99)00056-5.

[16] X. L. Xin, R. A. Borzooei, Y. B. Jun, Positive implicative soju ideals in BCKalgebras, Bulletin of the Section of Logic, vol. 48(1) (2019), pp. 1-18, DOI: https://doi.org/10.18778/0138-0680.48.1.01.

\section{Rajab Ali Borzooei \\ Shahid Beheshti University \\ Department of Mathematics \\ Tehran, Iran \\ e-mail: borzooei@sbu.ac.ir}

\section{Gholam Reza Rezaei}

University of Sistan and Baluchestan

Department of Mathematics

Zahedan, Iran

e-mail: grezaei@math.usb.ac.ir

\section{Mona Aaly Kologani}

Hatef Higher Education Institute Iran

Zahedan, Iran

e-mail: mona4011@gmail.com 


\section{Young Bae Jun}

Shahid Beheshti University

Department of Mathematics

Tehran, Iran

Gyeongsang National University

Department of Mathematics Education

Jinju 52828, Korea

e-mail: skywine@gmail.com 